\title{
Histochemistry of Acetylcholinesterase and Immunocytochemistry of an Acetylcholine Receptor-Like Antigen in the Brain of the Honeybee
}

\author{
SABINE KREISSL AND GERD BICKER \\ Institut für Neurobiologie, der Freien Universität Berlin, \\ 1000 Berlin 33 Federal Republic of Germany
}

\begin{abstract}
A histochemical staining method for acetylcholinesterase (AChE) and an antiserum raised against nicotinic acetylcholine receptors (AChR) of locust nervous tissue were applied in order to reveal certain candidates of cholinergic pathways in the brain of the honeybee. The AChE staining marked layers in the optic lobes, fibers connecting the two brain hemispheres, and fiber tracts as well as soma clusters within the protocerebrum. The calycal input regions of the mushroom bodies were labelled, whereas the intrinsic Kenyon cells showed no staining. Although the antennal afferents projecting into the dorsal lobe showed strong AChE activity, projections into the antennal lobe showed rather weak staining.

Application of the antiserum against the AChR showed immunoreactivity in neuropiles, tracts, somata, and the antennal nerve. The immunoreactivity of the optic lobes coincided with the banding pattern of the AChE staining. A particularly striking overlap of AChR immunoreactivity and AChE staining was found in the lip neuropile of the mushroom bodies, which would suggest a cholinergic input into this neuropile via fibers of the median antennoglomerular tract. Because the antiserum against locust AChR binds in neuropiles displaying AChE activity, we conclude that this antiserum also cross-reacts with the bee's receptor. This interpretation is supported by experiments showing $\alpha$-bungarotoxin ( $\alpha$-BTX) binding sites in some areas of strong immunoreactivity.
\end{abstract}

Key words: Apis mellifera, insect nervous system, antennal lobe, antennoglomerular tract, mushroom bodies

The central nervous system (CNS) of insects contains relatively large amounts of acetylcholine $(\mathrm{ACh})$, which is thought to serve as a neurotransmitter (Pitman, '85). The histochemical detection of cholinergic pathways can be achieved by mapping for one or more of the various components of the cholinergic system, such as the immunocytochemical detection of the ACh-synthesizing enzyme choline acetyltransferase (ChAT), autoradiographic studies of choline uptake, or the distribution of binding sites for radiolabelled ligands. Generation of an antiserum against an $\mathrm{ACh}$ conjugate (Geffard et al., '85a) and its immunocytochemical application to the insect brain of the locust has also been reported (Geffard et al., ' $85 \mathrm{~b}$ ). Because distribution of ChAT has been determined with an antiserum raised against the enzyme of this species (Lutz and Tyrer, '87), it should be possible to compare the localization of the transmitter with that of its synthetic enzyme.

Once released into the synaptic cleft, $\mathrm{ACh}$ binds briefly to its postsynaptic receptors before being hydrolyzed by acetylcholinesterase (AChE). We used a histochemical staining method for AChE (Karnovsky and Roots, '64) and an antiserum against acetylcholine receptors (AChR) of locust CNS (Breer et al., '85) in order to reveal certain candidates of cholinergic pathways in the brain of the honeybee. Studies on both the biochemical properties and the histochemistry of the bee's AChE have already been reported (Richards and Cutkomp, '45; Booth and Lee, '71; Booth and Metcalf, '72).

Accepted March 23, 1989 
According to Richards and Cutkomp ('45), the cholinesterase of bee brains is sensitive to eserine, and it hydrolyses, per mg per hour, four times as much $\mathrm{ACh}$ as butyrylcholine. Several modifications of the histochemical method for cholinesterase (Koelle and Friedenwald, '49) have been employed as a convenient method to localize cholinergic pathways. Because $\mathrm{AChE}$ is not confined exclusively to cholinergic neurons (Levey et al., '83), some caution is warranted in the interpretation of AChE activity as marker of a cholinergic pathway. For example, Kral and Schneider ('81) reported AChE activity in the photoreceptor axons of the worker bee, whereas Hardie ('87), on the basis of pharmacological investigations of the synapses between photoreceptors and monopolar cells, proposed histamine as a likely transmitter candidate of insect photoreceptors.

The present investigation addresses three issues: First, we describe AChE activity in defined tracts and neuropilar compartments, made possible by the increasingly well-documented anatomy of the bees's brain (e.g., Mobbs, '85). Second, this study complements earlier immunocytochemical studies on the localization of $\gamma$-aminobutyric acid (GABA) and glutamate, two other compounds that may also serve as classical transmitters in the nervous system of the bee (Bicker et al., '85; Schäfer and Bicker, '86; Bicker et al., '88). Third, in order to resolve some of the ambiguities in interpreting AChE histochemistry as an indicator of cholinergic pathways, we compared the distribution of enzymatic activity with the immunoreactivity of an AChR-like antigen. The monospecific antiserum was raised against an $\alpha$-BTX binding protein that represents a nicotinic $\mathrm{ACh}$ - receptor of the locust CNS (Breer et al., '85). The immunocytochemical application of the AChR antiserum to sections of locust and cockroach ganglia has already been described (Breer et al., '85; Breer and Sattelle, '87), showing strong immunoreactivity in the area of synaptic contacts in the neuropile, and less in the perikarial rind. Our comparison of AChE activity in identified tracts with the AChR-like immunoreactivity (AChR-LI) allows some conclusions about putative cholinergic pathways and their postsynaptic target areas in the brain of the bee.

\section{METHODS AChE histochemistry}

In order to locate AChE, we used the "direct-coloring" method, which employs acetylthiocholine iodide as substrate, for the detection of cholinesterases (Karnovsky and Roots, '64). The product of the cholinesterase activity, thiocholine, is believed to reduce ferricyanide to ferrocyanide, which precipitates as copper ferrocyanide directly at the site of enzymatic activity. Dissected brains were fixed overnight in a solution of $10 \%$ formalin containing $1 \% \mathrm{CaCl}_{2}$ dissolved in sodium phosphate buffer $(\mathrm{NaPi}, 0.1 \mathrm{M}, \mathrm{pH} 7.4)$, washed in phosphate-buffered saline (PBS) pH $7.4(0.9 \% \mathrm{NaCl} / 0,1 \mathrm{M}$ NaPi: $9 / 1$, and stored in $30 \%$ sucrose with $1 \%$ gum arabicum added. The tissue was blotted dry and quickly frozen in O.T.C. compound (Tissue Tek II, Miles) for sectioning. Serial sections $(16 \mu \mathrm{m})$ were cut in a cryostat and mounted on chrome alum-coated slides. Subsequently, they were processed according to the method described above and coverslipped. The sections were viewed and photographed with conventional optics.

\section{Immunocytochemistry}

A fixative composed of $4 \%$ paraformaldehyde dissolved in sodium phosphate buffer ( $\mathrm{NaPi}, 0.1 \mathrm{M}, \mathrm{pH} 7.4)$ was injected into the abdomens of live bees. The brains were subsequently dissected out of the head capsule and postfixed for 45 minutes in the same fixative. After rinsing in $\mathrm{NaPi}$, the tissue was dehydrated in increasing concentrations of ethanol and then infiltrated with paraffin. Serial sections, $10 \mu \mathrm{m}$ thick, were cut, deparaffinized, and processed according to the peroxidase-antiperoxidase method (PAP) (Sternberger, '79). Sections were incubated overnight at $4^{\circ} \mathrm{C}$ with the $\mathrm{AChR}$-antiserum in a dilution of $1: 200$ in PBS $(0.9 \% \mathrm{NaCl} / 0.1 \mathrm{M} \mathrm{NaPi}: 9 / 1,0.1 \%$ Triton X-100, $1 \%$ normal goat serum). Subsequently, the sections were rinsed in PBS and exposed to goat antirabbit IgG (Nordic), diluted 1:40, for 1 hour at room temperature. After another rinse with PBS, the sections were incubated at room temperature in the PAP complex (Dakopatts, diluted 1:100) for 1 hour. The sections then were washed in PBS $(0.9 \% \mathrm{NaCl} /$ $0.1 \mathrm{M} \mathrm{NaPi}$ : 9.1) and treated with 3,3'diaminobenzidine (DAB, Sigma, $25 \mathrm{mg} / 100 \mathrm{ml}$ ) and $\mathrm{H}_{2} \mathrm{O}_{2}(0.01 \%)$. Subsequently, they were dehydrated, cleared in xylene, and mounted in Entellan (Merck).

\section{Binding of $\alpha$-bungarotoxin}

Nervous tissue was fixed in $4 \%$ phosphate-buffered paraformaldehyde $(\mathrm{pH} \mathrm{7,4)}$ for 30 minutes, rinsed in PBS, quick frozen, and sectioned on a cryostat. Sections were incubated with $10^{-71} \mathrm{M} \alpha$-bungarotoxin dissolved in PBS overnight at $4^{\circ} \mathrm{C}$. At this concentration, the fluorescein isothiocyanate (FITC)-coupled bungarotoxin (Sigma) yielded an optimal staining of frog gastrocnemius neuromuscular junctions. After incubation, the sections were rinsed with PBS, mounted in PBS:glycerol 1:9 under coverslips, and the FITC fluorescence was photographed in a Reichert Polyvar microscope equipped with an appropriate filter combination (B1).

\section{RESULTS Specificity}

AChE-histochemistry. Unlike AChE, which is specific for $\mathrm{ACh}$, cholinesterases are enzymes that hydrolize a wide range of cholinesters (e.g., butyryl choline). Cholinesterases and $\mathrm{AChE}$ are therefore distinguished by using specific inhibitors and different substrates. Control experiments with butyrylthiocholine as substrate yielded only insignificant levels of labelling. Eserine (or physostigmine) is commonly used as an AChE inhibitor in insect neuropharmacology (Pitman, '85) and, when added to the reaction medium at a concentration of $10^{-4} \mathrm{M}$, blocks esterase activity completely (Fig. 1a). This provides further evidence that the histochemical staining is due to the activity of $\mathrm{AChE}$ and not other cholinesterases.

AChR-immunocytochemistry and $\alpha$-bungarotoxin binding. Omission of the primary antiserum in the immunocytochemical procedure resulted in a rather pale brown background staining. Immunocytochemistry with a nonimmune rabbit serum diluted 1:200 showed weak, homogeneous labelling of the sections (Fig. 1b). These observations rule out the possibility of artefacts from the immunocytochemical procedure such as endogenous peroxidase activity or nonspecific binding of the primary and secondary antisera. Because we could not perform preadsorption controls 

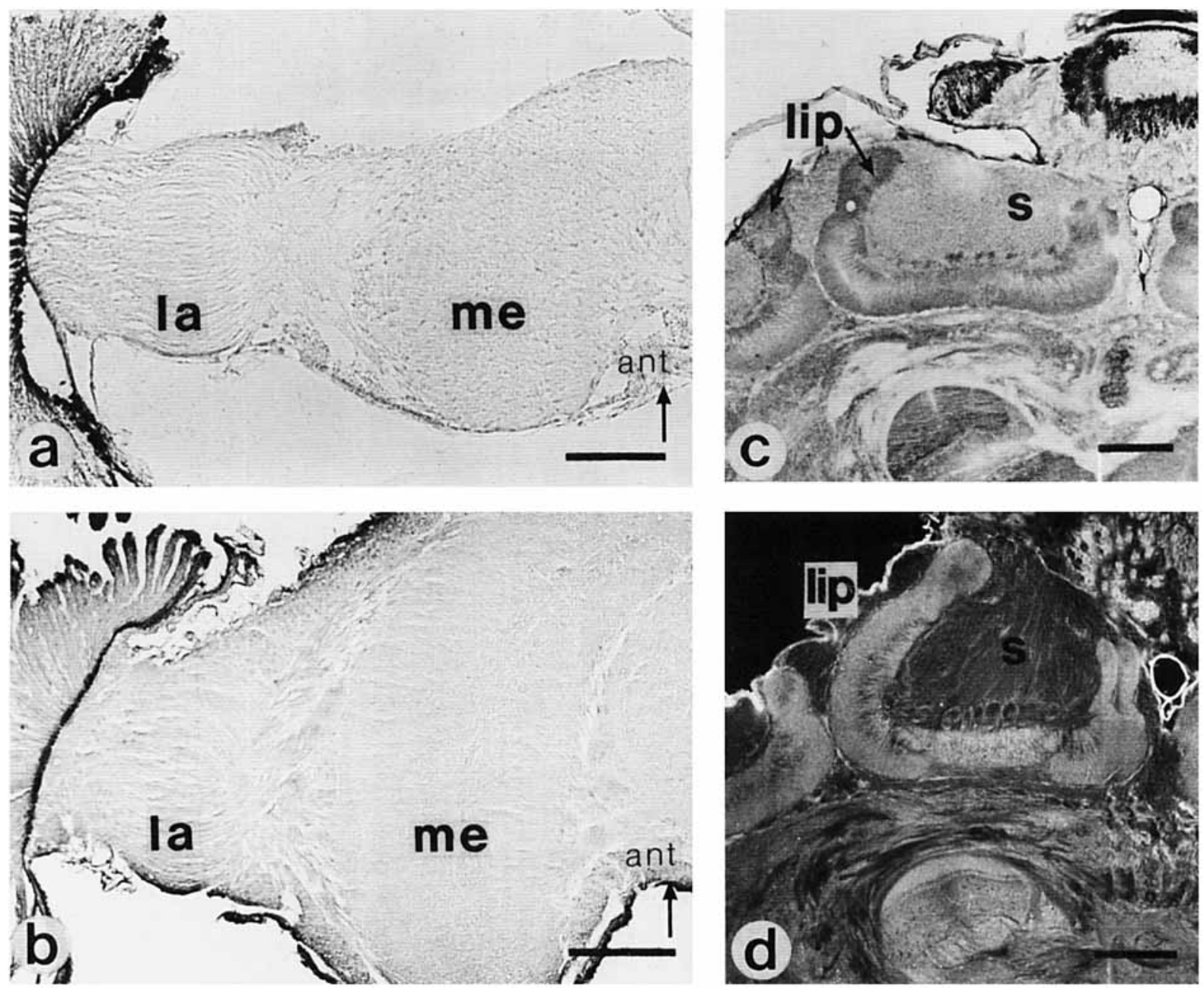

Fig. 1. Specificity of staining. a: AChE histochemistry on horizontal section. Incubation of section with eserine abolishes staining, demonstrating the sensitivity of the enzyme to an inhibitor specific for AChE. ant, anterior; la, lamina; me, medulla. b: AChR-immunocytochemistry on horizontal section. Incubation of sections with nonimmune serum yields only nonspecific background labelling. c: AChR-LI in frontal section of the mushroom bodies. Somata (s) of Kenyon cells are contained within the calycal cups of the mushroom bodies and show immunoreac-

of the AChR antiserum with the antigen, AChR-like immunoreactivity was compared with the distribution of $\alpha$-bungarotoxin ( $\alpha$-BTX) binding. In several areas where $\alpha$-BTX binding was indicated by FITC-fluorescence, for example, in the calyces of the mushroom bodies (Figs. 1c,d, Figs. 3b,d) or the layered neuropile of the optic lobes, we also detected strong AChR-LI. Because application of the AChR-antiserum in the PAP technique yielded a higher contrast than FITC-fluorescence, thus permitting better photographic reproduction, we relied mainly on the antiserum as a label for nicotinic AChR.

\section{Optic lobes}

The photoreceptors of the compound eyes provide the sensory input for three neuropilar masses with columnar tivity. AChR-LI is found in the calycal neuropile and is especially pronounced within the lip of the calyces. d: $\alpha$-BTX binding in frontal section of the mushroom bodies, as revealed by FITC fluorescence. Comparison with Figure 1c shows that the fluorescence is confined mainly to the calycal neuropile, whereas the somata of the Kenyon cells (s) show no labelling. Note that fluorescence is indicated by brightness of the micrograph, whereas immunoreactivity is indicated by darkening. Scale $\mathrm{a}-\mathrm{d}=100 \mu \mathrm{m}$.

organization: the lamina, medulla and lobula (Fig. 2). The majority of the retinula cell axons terminate in the most distal optic ganglion, the lamina, while three long visual fibers of each ommatidium project through the lamina and outer chiasma into a distal layer of the medulla (Ribi, '75). AChE activity was not detected between the basement membrane and fenestrated layer of the distal lamina (Fig. 3a), which indicates that axons of the photoreceptor cells do not display AChE activity.

The external plexiform C-layer, which is a narrow stratum closest to the outer chiasma, showed AChE activity (Figs. 2a, 3a), and this probably derives from amacrine interneurons projecting through the outer chiasma. This supposition is based on the fact that very fine $\mathrm{AChE}$ positive fibers, which are hardly resolved by our histochemical pro- 

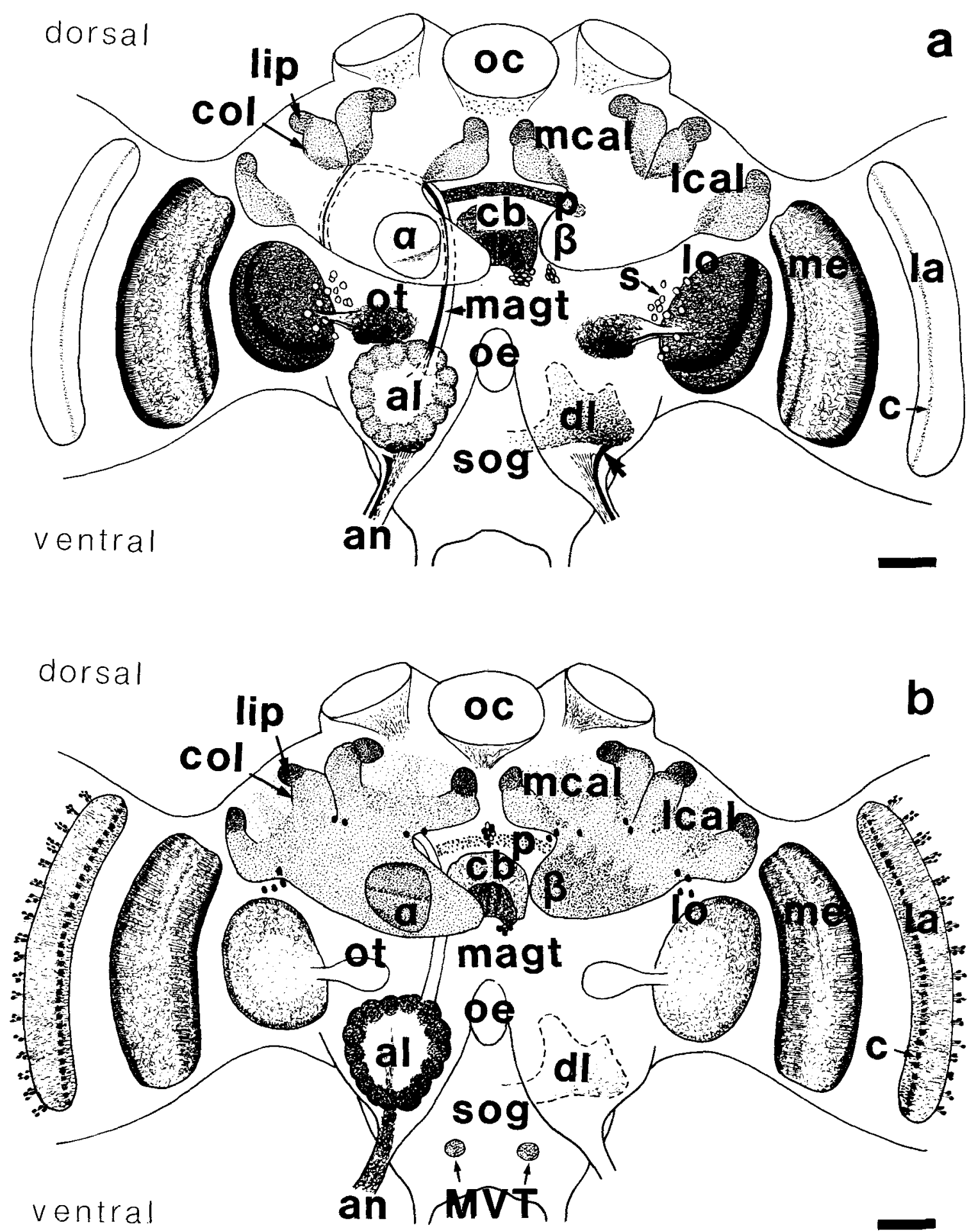

Fig. 2. Schematic drawings of labelling in the neuropiles and some prominent groups of somata in the bee's brain in a frontal view. a: AChE activity. The optic lobes lamina (la), medulla (me), and lobula (lo) display layered AChE staining. Weak AChE staining was found in the synaptic plexus of the ocelli (oc). The lobula is connected to the optic tubercle (ot). AChE-positive sensory fibers (arrow) of the antennal nerve project into the dorsal lobe (dl) and the suboesophageal ganglion (sog) below the oesophagus (oe). The median antennoglomerular tract (magt) connects the glomeruli of the antennal lobe (al) with the lip area (lip) of median (mcal) and lateral calyx (lcal) of the mushroom bodies. The collar (col) is a neuropilar compartment of the calyx receiving visual input. AChE positive fibers leave the $\beta$-lobe $(\beta)$ of the mushroom body. The central complex shows AChE activity in the pons ( $p$ ), central body (cb), and a group of somata (s) ventrally to the central body. C-layer (c), $\alpha$-lobe $(\alpha)$, antennal nerve (an). Scale $=100 \mu \mathrm{m}$. b: AChR-LI: The optic lobes lamina (la), medulla (me), and lobula (lo) display a layered AChRLI staining. The clustered somata of the monopolar cells in the lamina show strong AChR-LI. Sensory fibers of the antennal nerve (an) as well as the glomeruli of the antennal lobe (al) are intensely stained, whereas the dorsal lobe (dl) shows only weak immunoreactivity. The suboesophageal ganglion $(\mathrm{sog})$ contains an immunoreactive longitudinal sensory tract, the median ventral tract (MVT). All parts of the mushroom bodies are AChR-LI, including the somata of the Kenyon cells in the median (mcal) and lateral calyces (lcal). Similar to Figure $2 a$, the density of labelling is higher in the lip compared to the collar (col). The central body (cb) shows stronger immunoreactivity in the lower than in the upper division. Immunoreactivity is also found in the synaptic plexus of the ocelli (oc). magt, median antennoglomerular tract. Scale $=100 \mu \mathrm{m}$. 

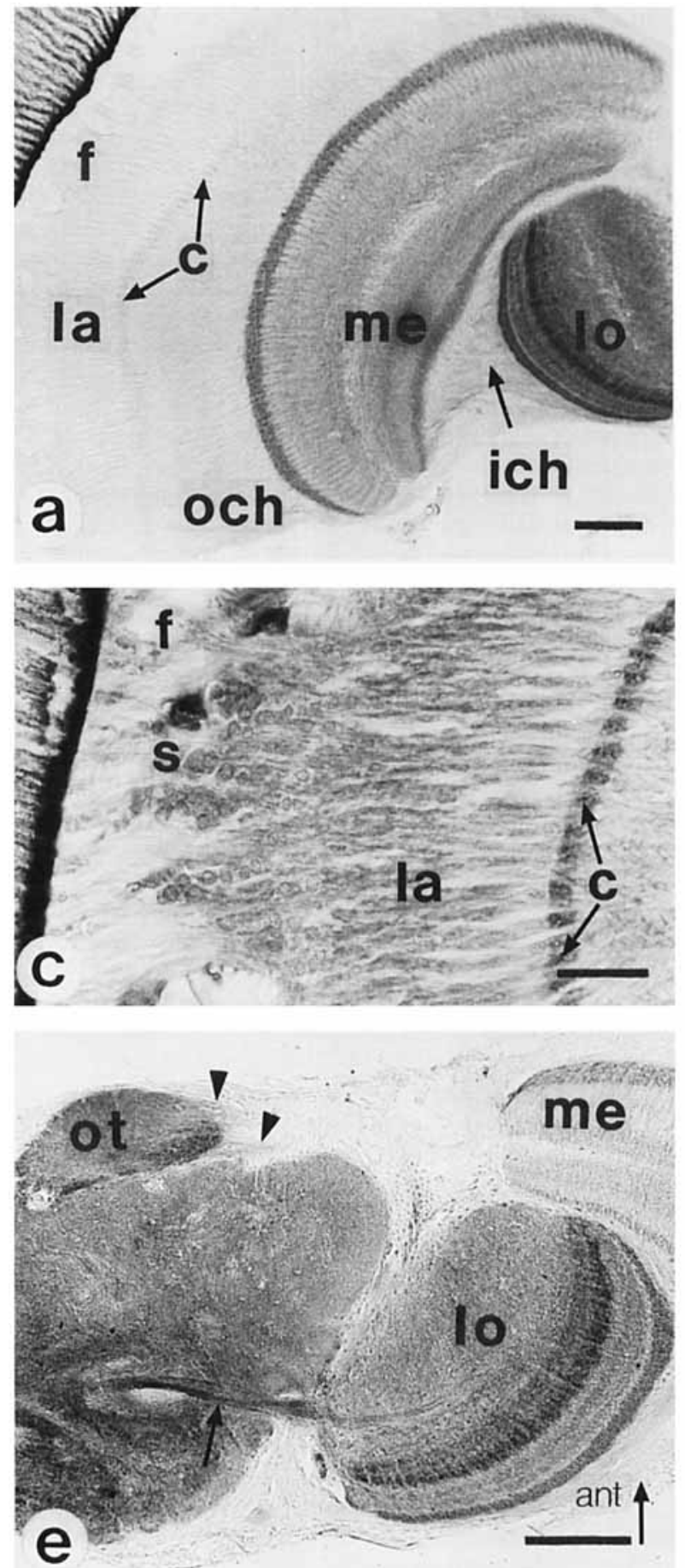

Fig. 3. Optic lobes. a: AChE histochemistry on frontal section of optic ganglia. Layers of AChE activity are found in the external plexiform c-layer (c) of the lamina (la), within the medulla (me), and lobula (lo). Stained fibers of small diameter cross through the outer (och) and inner chiasma (ich). The fenestrated layer (f) is not labelled. Scale $=100$ $\mu \mathrm{m}$. b: AChR-LI on frontal section of optic ganglia. The distribution of immunoreactivity in the c-layer of the lamina (la), the medulla (me), and the lobula (lo) corresponds to the layered $\mathrm{AChE}$ activity shown in Fig. 3a. AChR-LI in the lamina is not only confined to the c-layer but also found distally towards the fenestrated layer (f). Scale $=200 \mu \mathrm{m}$. c: AChR-LI on frontal section through the lamina. Somata (s), processes of monopolar cells, and the c-layer (c) are immunoreactive. Scale $10 \mu \mathrm{m}$. d:
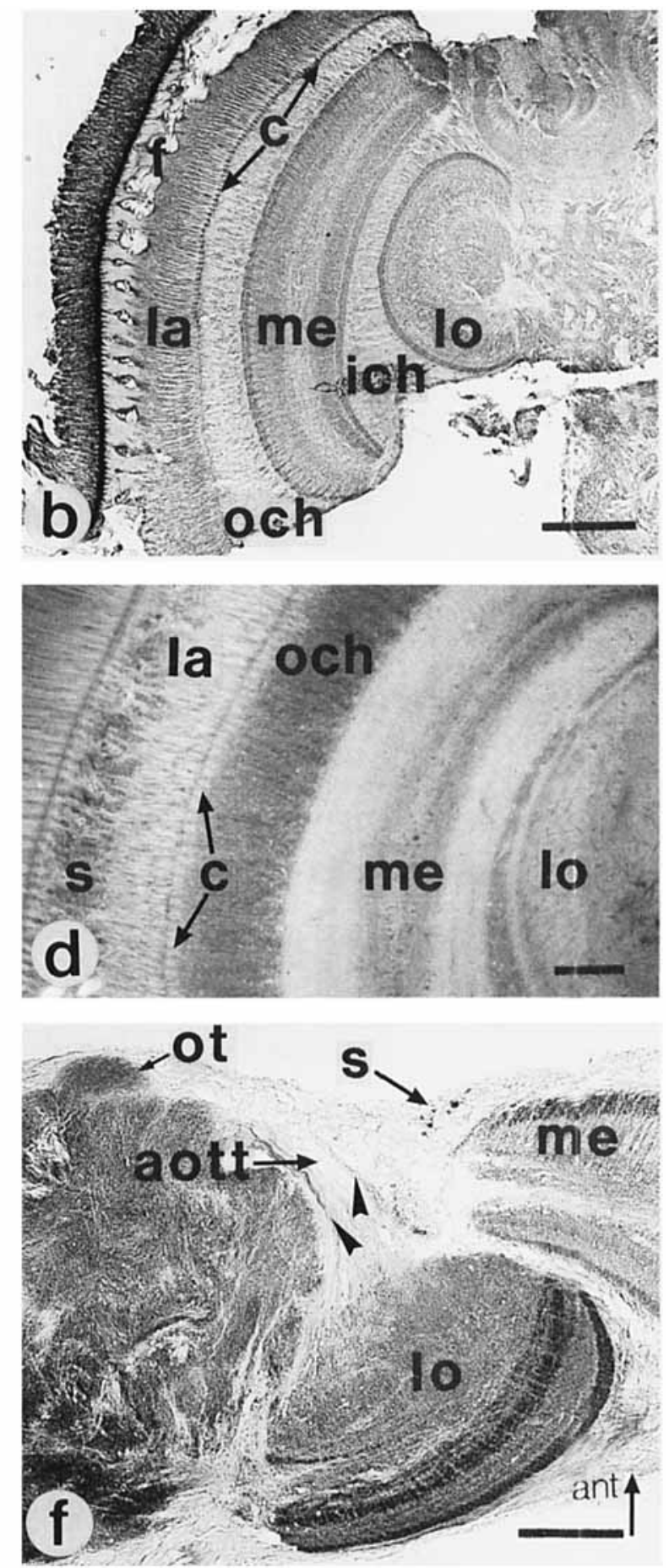

$\alpha$-BTX binding in frontal section of optic lobes, as revealed by FITC fluorescence. Note the similarity within the distribution of $\alpha$-BTX binding and AChR-LI (Fig. 3b). Fluorescence is indicated by bright areas within the micrograph, whereas immunoreactivity is represented by dark areas (Figs. 3b,c). Scale $=25 \mu \mathrm{m}$. e,f: $\mathrm{AChE}$ activity on two consecutive horizontal sections, showing stained fibers leaving the lobula (arrow). The planes of sectioning cut through the optic tubercle (ot), which is linked to the lobula (lo) through the anterior optic tubercle tract (aott). This tract contains AChE-positive fibers (arrowheads). Figure 3 reveals stained fibers within the inner chiasma and a group of labelled somata (s) anterior to the boundary of the medulla (me). Scale $=100$ $\mu \mathrm{m}$. 
cedure, approach the C-layer through the chiasma. Furthermore, in the close region of the medulla the chiasma is surrounded by weakly stained somata. The C-layer not only showed AChE activity but correspondingly exhibited AChR-LI (Figs. 2b, 3b). The fenestrated layer contains the somata of the first-order visual interneurons, the monopolar cells, which are clustered around tracheal invaginations (Fig. 2b). The monopolar cells showed strong AChR-LI, which was expressed at their surface, as well as a fine granular staining within the cytoplasm of their perikarya (Figs. $2 \mathrm{~b}, 3 \mathrm{c})$. The immunoreactivity in the $\mathrm{C}$-layer may be caused by the staining of lateral arborisations of the monopolar cells. Comparison of AChR-LI and $\alpha$-BTX binding (Figs. $3 \mathrm{c}, \mathrm{d})$ shows that the monopolar cells do not bind $\alpha$-BTX in the region of their perikarya, but that the C-layer and large portions of the lamina between fenestrated layer and $\mathrm{C}$ layer are labelled. The distribution of AChE activity, $\mathrm{AChR}-\mathrm{LI}$, and $\alpha$-BTX binding over the three optic ganglia is shown in Figures 3a,b,d. Because the strata forming the distal and proximal boundaries of the medulla and those of the posterior compartment of the lobula are labelled, there is a clear overlap of AChE-activity, AChR-LI, and $\alpha$-BTX binding. The most distal AChE-stained layer of the medulla sends fine, evenly spaced invaginations into underlying layers (Figs. 2a, 3a). The organized spacing of these extensions suggests that local interneurons, which are organized in columns, contribute to the layered appearance of the medulla in the histochemical staining. The central parts of the medulla display rather low staining, especially within the area of the serpentine layer.

The medulla is linked to the lamina via the outer chiasma, which contains very thin AChE-positive fibers that were hardly resolved by our method. The inner chiasma between the medulla and lobula shows AChE activity, which may result from a columnar fiber projection between the two visual neuropiles. Because the strata of the lobula are tilted toward the frontal plane, layering of AChE activity in the lobula can best be visualized in a horizontal plane of section (Figs. 3e,f). Such sections demonstrate the intensely stained layer 1 (Ribi and Scheel, '81) at the most posterior face of the lobula and a centrally located second band corresponding to layers 3 and 4 . The anterior neuropilar compartment of the lobula does not exhibit the columnar organisation of the visual system and contains more diffuse arborisations of AChE-positive fibers.

Both labelled and unlabelled unidentified fibers connect the lobula with the protocerebral and deutocerebral parts of the brain (Figs. 3e,f, $4 \mathrm{c}, \mathrm{d}$ ). The anterior optic tubercle tract connects the optic tubercle, a glomerular structure lying above the antennal lobe, with the lobula (Fig. 2). Some fibers at the margins of the anterior optic tubercle tract to lobula displayed AChE staining (Fig. 3f), and the entire neuropile of the optic tubercle was labelled (Figs. 3e, 5a). Several commissures connecting the optic ganglia of the two brain hemispheres (Mobbs, '85; Hertel and Maronde, '87) contained AChE-stained fibers. In the dorsal fiber population of the anterior optic tract, a small percentage of fibers was labelled (Figs. 4a,b). Among other fibers, the anterior optic tract contains projections from the anterior superior optic tract that extend from the dorsal medulla and lobula into the outer ring tract of the calyces. Several fibers of the anterior superior optic tract that pass dorsally to the pedunculus display AChE activity (Fig. 6a). The ventral posterior optic commissure crosses above the esophagus in the most posterior part of the protocerebrum and links the two lobu- lae. About one third of the fibers within this commissure show AChE activity (Figs. 4b,d).

An identified single pair of movement-sensitive neurons has been described (DeVoe et al., '82; Hertel et al., '87) that more than likely correspond to the lobula extrinsic AChEstained fiber of Fig. 4c. This characteristic cell arborises in layers 4 and 5 of both lobulae, with the position of the soma lateral to the optic tubercle. Its axon passes behind the optic tubercles and follows the interoptic tubercle tract to the other side of the brain. AChE staining revealed the arborisations of this cell in the layers of the lobula, and the anteriorly looping neurite could be traced up to a position behind the optic tubercle (Fig. 4c). Furthermore, sagittal serial sections showed the two axon profiles close to the intertubercle tract (Fig. 6d).

A major difficulty in analysing the shape and identity of neurons containing AChE activity was that the connection between perikaryon and the main neurites through the thin primary neurite could not be resolved satisfactorily. Therefore, in many cases, the correspondence between the location of stained groups of somata and their neuropilar processes remained unclear. Two rather prominent groups of about 30 distinct, heavily stained somata are located anterior to the boundary of each lobula (Figs. 2a, 3f). Unfortunately, their axons could not be traced as mentioned above.

\section{Ocellar tract neuropile}

The honeybee possesses three dorsal ocelli in addition to the compound eyes (Fig. 2). The ocellar photoreceptor cells synapse within the ocellar plexus onto 30 large (L-neurons) and approximately 80 small (S-neurons) interneurons (Goodman, '81). Neither AChE activity nor AChR-LI was detected within the photoreceptors or the L-neurons (Fig. 4b). However, weak AChE activity, strong AChR-LI, and also $\alpha$-BTX binding was found within the neuropile of the ocellar plexus (Fig. 2b). Because neither photoreceptors nor L-neurons displayed AChE activity, it is likely that this resulted from a subpopulation of S-neurons that invade the neuropile of the ocellar plexus.

\section{Antennal and dorsal lobe}

Antennal and dorsal lobes are deutocerebral parts of the insect brain. The antennal lobes receive their principal sensory input from chemosensory receptors on the antennae, whereas antennal mechanosensory fibers project mainly into the dorsal lobes (Pareto, '72; Suzuki, '75). In the antennal lobe, sites of synaptic contacts between receptor terminals, local interneurons, and output relay interneurons are arranged as spherical glomeruli surrounding the central neuropile (Matsumoto and Hildebrand, '81; Boeckh et al., '84; Arnold et al., '85; Homberg, '84). Both the antennal and the dorsal lobes showed AChE activity (Fig. 2a). In the antennal lobe, activity was confined mainly to the glomeruli (Figs. 5a,b), whereas the central neuropile was not stained. A saggital section through the brain (Fig. 5a) demonstrates that the glomeruli are clearly stained in comparison to the unlabelled pedunculus of the mushroom body, but that the egg-shaped dorsal lobe contains fibers showing a higher level of AChE activity than the antennal lobe. The sensory fibers of the antennal nerve, which project ventrally to the antennal lobe into the dorsal lobe, contribute largely to the AChE staining of the dorsal lobe neuropile (Figs. 5a,b), whereas the sensory projections into the antennal lobe show only a rather weak staining. Some glomeruli receiving 

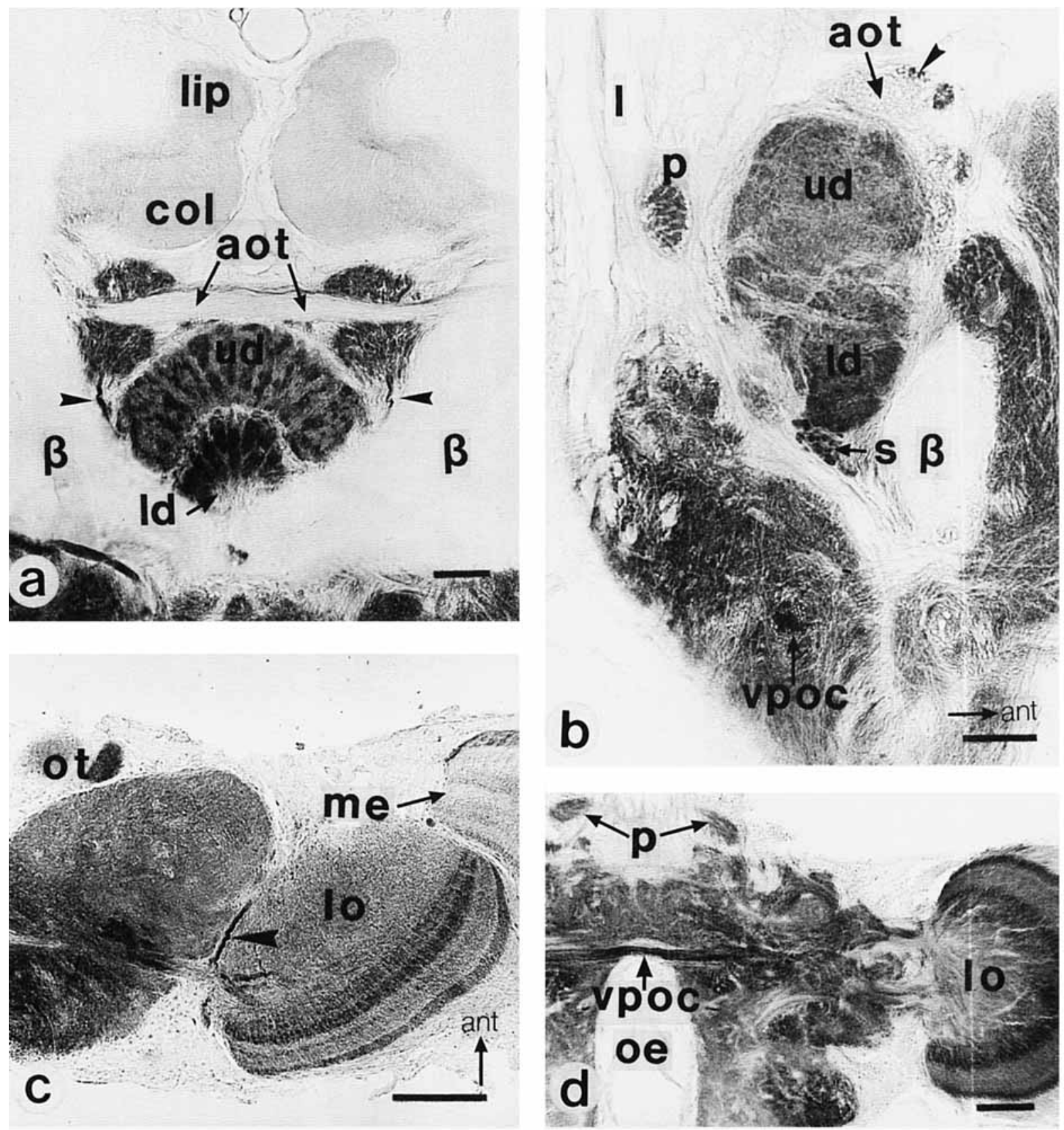

Fig. 4. AChE activity in commissures of the brain hemispheres. a: Frontal section through the protocerebrum at the level of the anterior optic tract (aot), which contains stained fibers in its dorsal portion. The stained central body, with its upper division (ud) and lower division (ld), is flanked by two labelled fibers (arrowheads). In the neuropile of the mushroom bodies, AChE activity can be seen only in the lip and collar (col) of the calyces, but not within the $\beta$-lobes $(\beta)$. Scale $=50 \mu \mathrm{m}$. b: Sagittal section close to the midline through the protocerebrum. A stained cluster of somata $(\mathrm{s})$ is situated ventrally to the central body. The lower division (ld) is more strongly labelled compared to the upper division (ud). AChE activity is evenly distributed through the crossection of the

pons (p), but only found in certain fibers (arrowhead) of the anterior optic tract (aot), and the ventral posterior optic commissure (vpoc). There is no staining in the $\beta$-lobe ( $\beta$ ) and the ocellar L-neurons (l). Scale $50=\mu \mathrm{m} . c$ : Horizontal section showing the arborisations of a stained fiber (arrowhead), which exits the lobula (lo) in an anterior direction towards the optic tubercle (ot) and crosses into the contralateral brain hemisphere close to the intertubercle tract. me, medulla. Scale $=100$ $\mu \mathrm{m}$. d: Frontal section showing AChE positive fibers in the ventral posterior optic commissure (vpoc), and stained as well as unstained fibers in the lobula (lo). The section also contains the most distal portions of the pons (p). oe, oesophagus. Scale $=100 \mu \mathrm{m}$. 

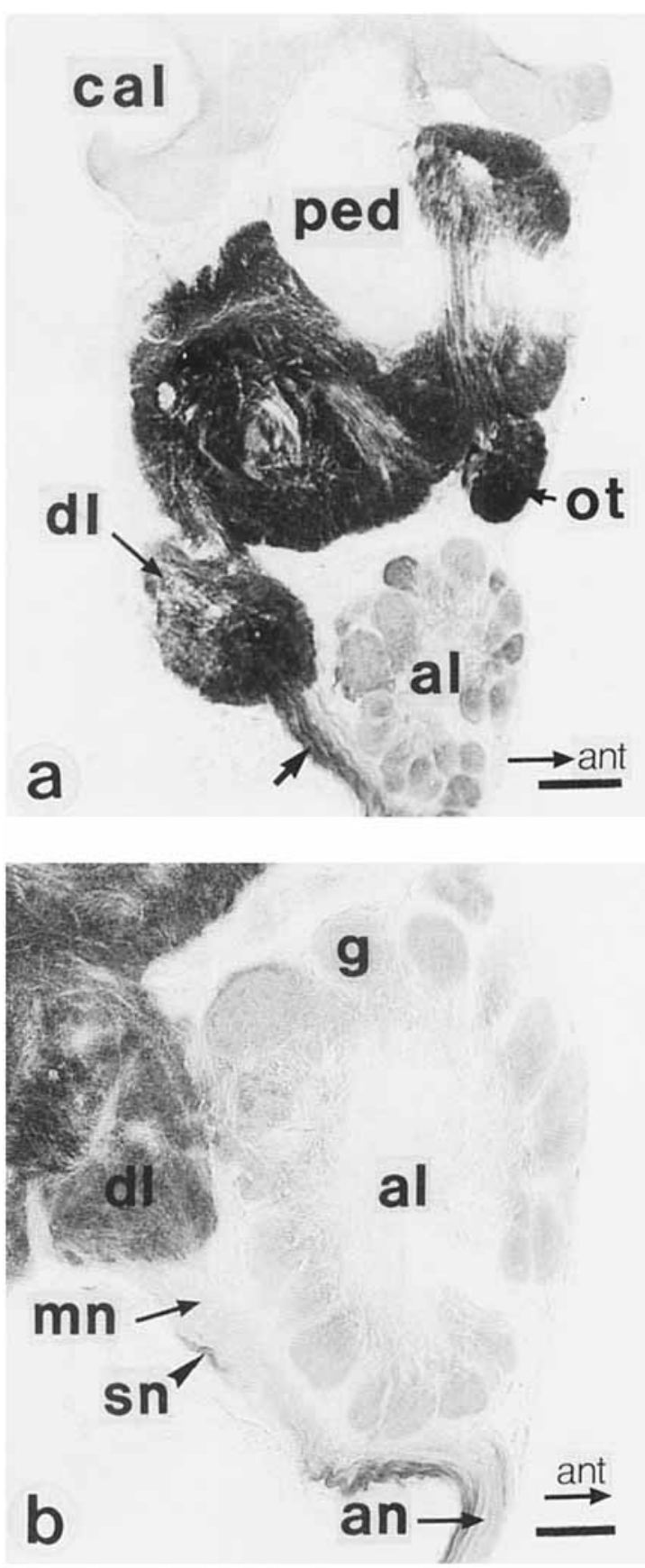

Fig. 5. Sensory projections from antennae, head hairs and mouth nerves into the deutocerebrum and suboesophageal ganglion. a: Overview of $\mathrm{AChE}$ activity on a sagittal section through the brain at the level of the antennal lobe (al), dorsal lobe (dl), and optic tubercle (ot). The mushroom bodies show staining in the calyces (cal) but not in the pedunculus (ped). Sensory afferents to dorsal lobe (arrow). Scale $=100$ $\mu \mathrm{m}$. b: Sagittal section through antennal lobe (al). Sensory neurons (sn) of the antennal nerve (an) projecting into the dorsal lobe (dl) show stronger AChE staining than sensory projections into the glomeruli (g) of antennal lobe. Motoneurons $(\mathrm{mn})$ originating in the dorsal lobe are not stained. Scale $=50 \mu \mathrm{m}$. c: AChR-LI on sagittal section through the antennal lobe (al) and suboesophageal ganglion (sog). Strong immunoreactivity is found in the antennal nerve (an) and its sensory projections into the glomeruli. The $T_{1}$ tract is an example of a sensory fiber bundle
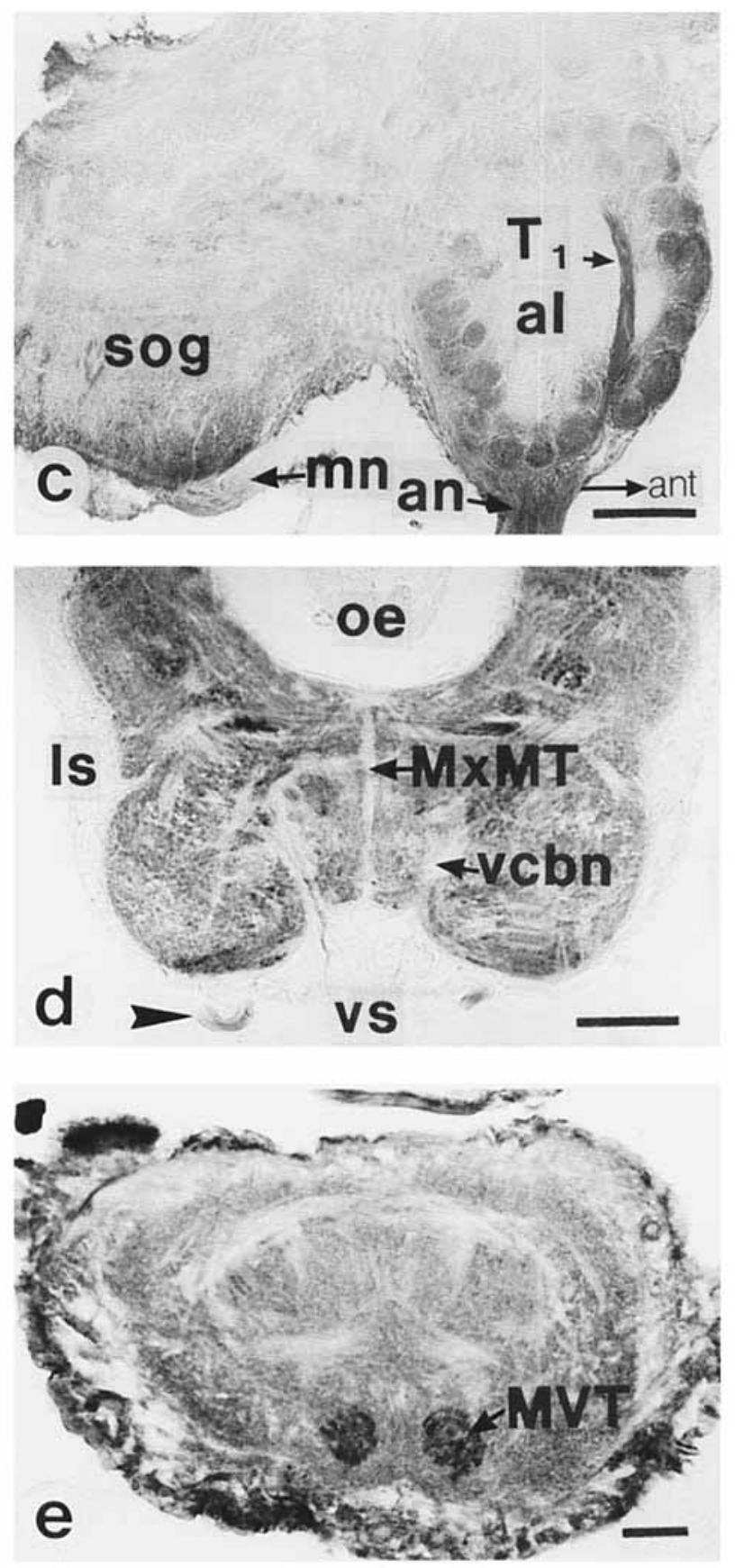

that supplies the dorsal group of glomeruli. The mandibular nerve (mn) of the sog shows fewer immunoreactive fibers than the completely stained antennal nerve (an). Scale $=100 \mu \mathrm{m}$. d: AChE activity on a frontal section through the suboesophageal ganglion. Arrow delineates stained fiber in ventral cell body neurite tract (vcbn). Although many tracts, for example, the maxillary midline tract (MxMT), and also the lateral (ls) and ventral soma layer (vs) are not labelled, the neuropile is penetrated by AChE-positive fibers. Stained sensory neurons are found in the maxillary nerves (arrowhead). oe, oesophagus. Scale $=100 \mu \mathrm{m}$. e: AChR-LI on a frontal section through the labial neuromere of the suboesophageal ganglion, showing immunoreactivity in soma rind and neuropile. The strongest labelling is found within the median ventral tract (MVT). Scale $50 \mu \mathrm{m}$. 

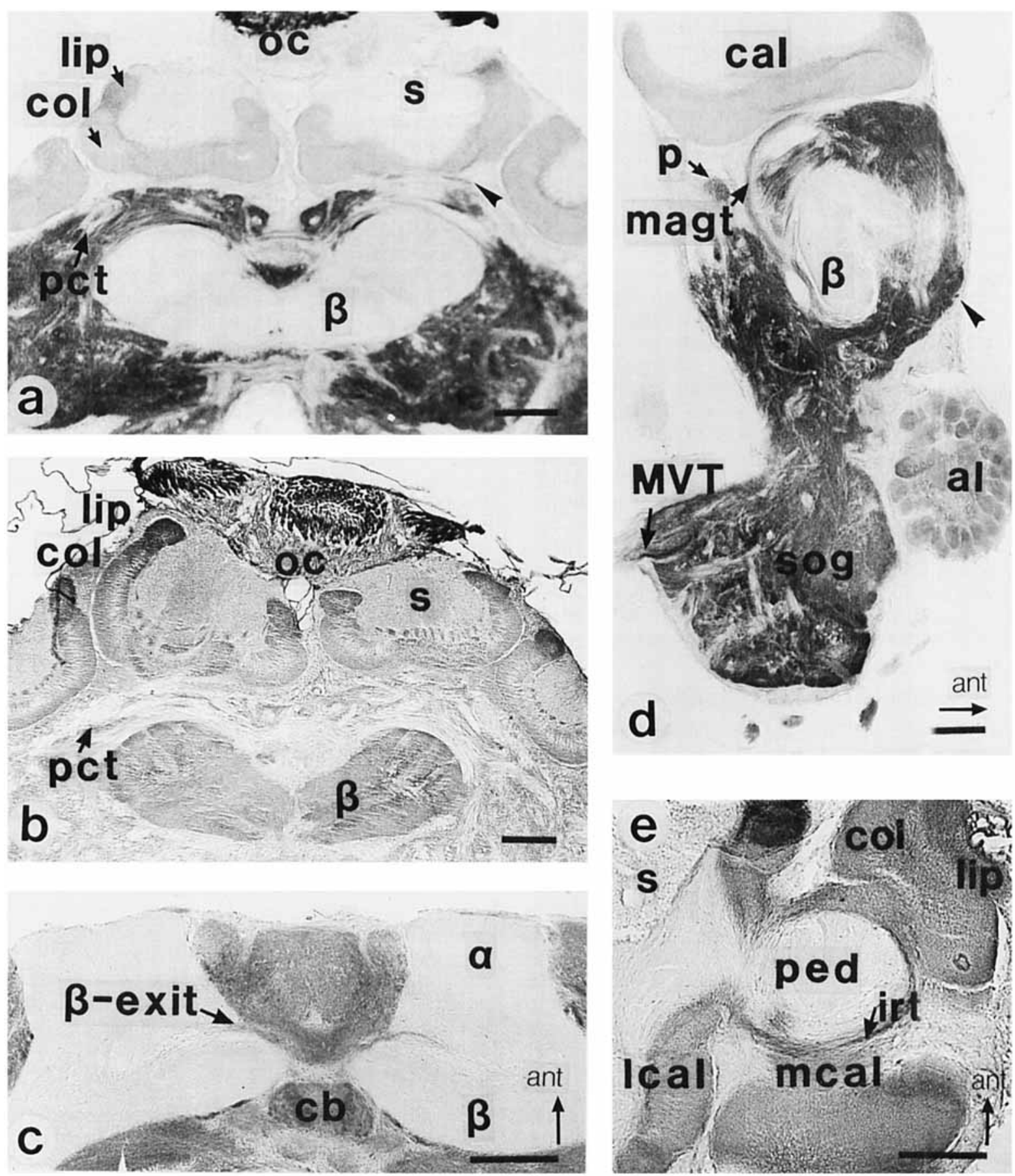

Fig. 6. Protocerebral pathways. a: Distribution of AChE activity on frontal section of protocerebrum. Dense staining is found in the neuropile surrounding the mushroom bodies; note the difference in the staining intensity between lip and collar (col) of calycal neuropile. Arrowhead delineates stained fibers of the anterior superior optic tract. The protocerebro calycal tract (pct) is not labelled. The ocellar plexus ventrally to the ocelli (oc) is weakly stained. Somata (s) of Kenyon cells are not labelled. Some weakly stained fibers are found within the pedunculus dorsally to the $\beta$-lobes $(\beta)$. b: Distribution of AChR-LI on frontal section of protocerebrum. Somata (s), calycal neuropile, and lobes of the mushroom bodies are labelled. The highest density of staining is found in the lip of the calyces (compare with Fig. 5a). The protocerebro calycal tract (pct) is not stained. c: Distribution of $\mathrm{AChE}$ activity on horizontal section through the $\alpha$-lobe $(\alpha)$ and $\beta$-lobe $(\beta)$ of the mushroombodies. A few stained extrinsic fibers project through the $\beta$-exit. Central body (cb). d: Distribution of AChE activity on sagittal section of the brain and the suboesophageal ganglion (sog). The median antennoglomerular tracts (magt) links the antennal lobe (al) with the calyces (cal) and contains stained fibers. The pons (p), in the posterior protocerebrum is evenly labelled. Arrowhead delineates the crossection of the two heterolaterally projecting interlobula neurons. The $\beta$-lobe $(\beta)$ and several tracts and commissures in the sog are not stained, whereas the longitudinally projecting median ventral tract (MVT) is stained. e: Distribution of AChE activity on a horizontal section through the median (mcal) and lateral calyx (lcal). Stained fibers of the median antennoglomerular tract enter the calycal neuropile via the inner ring tracts (irt) and project into the lip. ped, pedunculus. Scale a-e $=100 \mu \mathrm{m}$. 
strongly AChE-stained fibers from the antennal nerve, however, were observed at the entrance of the nerve into the lobe. The dorsal lobe is the origin of those motoneurons responsible for the movement of the antenna (Pareto, '72; Suzuki, '75). Their large-diameter axons, which do not contain AChE activity, can be seen leaving the dorsal lobe (Fig. $5 b)$. Degeneration and dye backfilling studies of the antennal nerve (Pareto, '72; Suzuki, '75; Mobbs, '85; Arnold et al., '85) have also revealed direct sensory projections into the posterior deutocerebral and tritocerebral parts of the brain, as well as into the suboesophageal ganglion (sog). These projections could be traced into the sog and their AChE activity identified.

A large density of AChR-LI was found in the antennal nerve and the glomeruli of the antennal lobe (Figs. 2b, 5c). Once again, most staining within the lobe was confined to the glomeruli, where synaptic interactions are thought to occur. Various strands of sensory fibers passing through the central neuropile also exhibit the AChR-LI. Figure $5 \mathrm{c}$ shows AChR-LI in the antennal nerve and the T1 strand (Mobbs, '85) of the sensory fibers. The strong immunoreactivity is peculiar to the antennal nerve, as, for example, the mandibular nerve (Fig. 5c) did not show the high density of AChRLI. Although motoneurons of the suboesophageal ganglion ( $\mathrm{s} \circ \mathrm{g}$ ) and the dorsal lobe may show AChR-LI at the surface of their arborisations, AChR-LI was not detected within their axons. The brain of the bee contains groups of somata that display AChR-LI to a greater extent than that of neighbouring somata. Such a group of unidentified AChR-LI somata is positioned in the mediodorsal soma rind of the antennal lobe.

\section{Suboesophageal ganglion}

The sog is contiguous with the deutocerebral and tritocerebral parts of the brain and arises from the fusion of the mandibular, maxillary, and labial neuromere. There are not only direct sensory projections from the antenna to the suboesophageal ganglion, but also fibers from the wind-sensitive head and interommatidial hairs (Neese, '65). Sensory axons are collected within the tegumentary nerve, which projects along the posterior face of the brain into the suboesophageal and prothoracic ganglia. Three-quarters of the fibers terminate within the tritocerebrum and sog, and the remainder descend to the prothoracic ganglion (Mobbs, '85). Fibers of the tegumentary nerve showed AChE activity. Within the suboesophageal ganglion, these fibers join other sensory fibers of the mouth nerves within the median ventral tract (Rehder, '88).

Like those of the antennal sensory projections, the fibers of the median ventral tract are strongly AChR-LI immunoreactive (Figs. 2b, 5e). Both AChE activity and AChR-LI were distributed within the neuropiles of the sog (Figs. 5d,e, $6 d)$. A prominent tract containing descending neurons of the brain is the median dorsal tract (MDT), which extends through the sog before it joins the cervical connective. None of the large-diameter neurons within this tract showed AChE activity. The sog innervates various mouthparts via several nerves. The large-diameter fibers within these nerves were not stained, but the small diameter sensory fibers showed AChE activity. The neuropile and many tracts and commissures contained AChE-stained fibers. However, because hardly any stained somata were detected in the soma rind of all three neuromeres, the majority of the labelled neurons must originate outside of the sog. For example, only a single labelled fiber could be found in a tract containing primary neurites of ventral cell bodies (vcbn; Rehder, '88) of the maxillary neuromere (Fig. 5d).

\section{Mushroom bodies}

The relay neurons of the antennal lobe project via the median, lateral, and mediolateral antennoglomerular tracts (magt, lagt, and mlagt) into the ipsilateral protocerebrum. They eventually terminate in or near the calyces of the mushroom bodies and projection areas of the lateral deutocerebral neuropile area (Mobbs, '85) (Figs, 2a,b). The calycal neuropile can be subdivided into lip, collar, and basal ring regions. Antennal relay neurons enter the calyx via the inner ring tract and terminate within the lip, whereas the collar receives mainly input from the optic ganglia via the outer ring tract. AChE staining of the mushroom bodies showed a clear compartmentalized pattern. Although the somata and neurites of the mushroom-body intrinsic Kenyon cells were not stained, the calycal neuropile showed AChE activity (Figs. 4a, 5a, 6a,d). AChE activity is especially pronounced in the lip area and can be attributed to fibers of the magt (Fig. 6d), which approach the lip area through the inner ring tract (Fig. 6e). AChE activity is not homogeneously distributed across the fiber population of the magt, but the fibers at the outer flanks of the tract exhibit strong AChE activity, whereas fibers in the core showed intermediate or no staining. Nevertheless, AChE activity in a subpopulation of magt fibers seems sufficient to explain the staining of the lip. The anterior superior optic tract, which carries projections into the collar, contained a few stained fibers (Fig. 6a), and these fibers may be responsible for the staining. Labelling in the basal ring, which receives projections from the tritocerebrum and sog, according to Mobbs ('85), is unaccounted for. Apart from the calyces, most parts of pedunculus and lobes are devoid of staining. Some extrinsic fibers which leave the mushroom body neuropile through the $\beta$-exit did, however, show $\mathrm{AChE}$ activity (Fig. 6c).

All parts of the mushroom body show AChR-LI. The immunoreactivity in the calyces reaches its greatest density in the lip area, which shows also the strongest $\mathrm{AChE}$ activity (Figs. 2a,b). It is difficult to separate the contribution of mushroom body intrinsic and extrinsic fibers to the immunoreactivity. Because the somata within the calyces (Fig. 6b) contain granular immunoreactivity and all neuropilar compartments of the mushroom body show strong, but fine immunoreactivity, the Kenyon cells express AChR-LI. On the other hand, prominent tracts connecting to the mushroom body neuropile, like the protocerebro calycal tract (Fig. 6b) or the magt, did not show AChR-LI within their axons.

Several somata showing AChR-LI were found in the lateral vicinity of the calyces (Fig. 2b). Another cluster was found in the anterior space between the median and lateral calyx, and one prominent cluster was observed near the central trachea.

\section{Central complex}

The central complex in the midbrain is comprised of the central body, the two glomerular noduli, and the protocerebral bridge (pons) (Fig. 2). AChE activity is distributed in both the lower and upper division of the central body, but it covers a larger part of the neuropile in the lower division (Fig. 4a,b). Correspondingly, the AChR-LI in the lower division appears more dense than in the upper division. AChEstained fibers were found approaching the lower division 
through the isthmus tracts (Homberg, '85). Projections into the upper and lower division show the typical fan-shaped pattern, which is characteristic of innervation of the central body. The central body is connected to the noduli and protocerebral bridge (pons), which show a homogenous distribution of AChE activity as well as AChR-LI. On both sides, the central body is flanked by a single AChE-stained fiber (Fig. 4a) that arborises in the neuropile surrounding the anterior optic tract. Another conspicuous feature of the central body is a bilateral cluster of strongly AChE-stained somata located ventrally to the lower division (Fig. 2a, 4b).

\section{DISCUSSION Antennal afferents and specificity of staining
for cholinergic pathways}

This paper provides a detailed account of the distribution of AChE activity in an insect brain. Because application of the histochemical staining method of Karnovsky and Roots ('64) to the bee's brain proved to be sensitive to the inhibitor eserine, and control experiments with butyrylthiocholine as substrate yielded only insignificant levels of labelling, it is likely that the staining is specific for AChE. The reported histochemical data also support the biochemical measurements (Richards and Cutkomp, '45) that demonstrated the sensitivity of the bee's AChE to eserine and its higher specificity for ACh compared with butyrylcholine.

The specificity of the staining for AChR-LI is rather difficult to establish. In the locust and cockroach, the monospecific antiserum stains areas of the neuropile receiving cholinergic input and, in addition, recognizes an $\alpha$-BTX binding protein of $65 \mathrm{Kd}$ on immunoblots of locust nervous system (Breer et al., '85; Breer and Sattelle, '87). Because of the limited availability of the antigen and antiserum, we could not perform preadsorption controls and immunoblots with nervous tissue of the bee. Nevertheless, the overlap of AChR-LI, AChE activity, and $\alpha$-BTX binding is striking in very distinct areas of layered neuropiles, e.g., the optic lobes (Figs. 2, 3a,b,d). Thus, we were able to evaluate independent evidence for cholinergic neurotransmission in certain parts of the brain.

In insects, the chemical neuroanatomy of the cholinergic system is particularly well documented for Drosophila, where AChE activity (Hall and Kankel, '78), ChAT-immunoreactivity (Salvaterra et al., '85; Buchner et al., '86; Gorczyca and Hall, '87), choline uptake (Buchner and Rodrigues, '83), and $\alpha$-BTX binding sites (Schmidt-Nielson et al., '77; Rudloff, '78; Dudai, '80) have been investigated. Although not congruent in every instance, various indicators of cholinergic pathways did show considerable overlap. Owing to its high concentration, $\mathrm{ACh}$ has been suspected as a major neurotransmitter of the insect brain (e.g., Breer, '81), but physiological evidence for its transmitter action has so far been restricted to the sensory afferents of the antennae. Biochemical studies of the level of ACh content and its metabolic enzymes during development of the antennae in the moth Manduca have led to the hypothesis that the antennal sensory neurons are cholinergic (Sanes and Hildebrand, '76). This was supported by mapping $\alpha$-BTX binding sites in normal and deafferented antennal lobes (Hildebrand et al., '79). Further evidence comes from an immunocytochemical study performed in the locust (Lutz and Tyrer, '87), in which ChAT-immunoreactive fibers entering the antennal lobe from the antennal nerve were described.
In the bee, antennal sensory afferents projecting into the dorsal lobe, the subeosophageal ganglion, and the posterior deutocerebrum did indeed show strong $\mathrm{AChE}$ activity, whereas staining of the sensory projections into the glomeruli of the antennal lobe was rather weak. The functional significance of the striking biochemical diversity in $\mathrm{AChE}$ activity between antennal and dorsal lobe afferents of the bee remains unknown. Some evidence points toward a mechanosensory role of the dorsal lobe, as it receives projections from the antennal chordotonal organ and tactile sensilla positioned at the base of the scapus (Pareto, '72); antennal lobe afferents are thought to carry chemosensory information. The high level of $\mathrm{AChE}$ activity in the projections leading to the dorsal lobe supports the cholinergic nature of these fibers, whereas the low level of AChE activity in the antennal lobe projections raises some doubt about the cholinergic status of the chemosensory fibers. A recent immunocytochemical study on the distribution of $\mathrm{ACh}$ in the crayfish brain provided evidence for $\mathrm{ACh}$ immunoreactivity only in the statocyst, but not the chemoreceptor afferents of the antennulary nerve (Wang-Bennet et al., '88). In the bee's antennal nerve, it is also possible that both types of sensory fibers use $\mathrm{ACh}$ as transmitter, but that some unknown constraints in the processing of different sensory modalities may require different levels of AChE activity in the neuropile of the dorsal lobe and the glomeruli of the antennal lobe.

The molecular diversity between dorsal lobe and antennal lobe afferents is also reflected in the AChR-LI, which is strongly expressed in the antennal lobe afferents and to a much lesser extent in the dorsal lobe afferents. Several possibilities may explain the AChR-LI in the antennal lobe afferents. First, the antennal lobe afferents may receive efferent cholinergic input and therefore may express a nicotinic receptor. Second, based on the assumption that $\mathrm{ACh}$ is the sensory transmitter, the AChR immunoreactive antigen in the antennal afferents may represent a presynaptic autoreceptor. Pharmacological investigations of the intracellularly recorded lateral filiform hair sensory neurone in the first instar cockroach provide evidence that nicotinic presynaptic or extrasynaptic $\mathrm{AChR}$ are present on the cholinergic sensory axon (Blagburn and Sattelle, '87).

Certainly, the present immunocytochemical study cannot rule out a third remote possibility, i.e., besides staining nicotinic AChRs, as suggested by the overlap of immunoreactivity, $\alpha$-BTX binding, and AChE activity in some neuropiles, the polyclonal antiserum against locust receptor labels some unknown additional antigens of bee neurons. If this explanation is taken for granted, it should be noted that these antigens are shared not only by the sensory afferents of the antennal lobe, but also by all sensory projections from the mouth nerves and the tegumentary nerve into the sog and projections running in the median ventral tract (Figs. 2b, $5 e)$. Because the tegumentary nerve of the locust shows ChAT immunoreactivity (Lutz and Tyrer, '87), these sensory afferents are also candidates of cholinergic cells; however, because these unknown antigens seem associated with putative cholinergic pathways, we favour the interpretation that the immunoreactivity is indeed caused by nicotinic AChRs.

\section{Optic lobes and ocellar system}

Other parts of the brain, such as the layered optic lobes, show a clearer correspondence between AChE activity, AChR-LI, and $\alpha$-BTX binding (Figs. 3a,b,d). The staining 
in the lamina provides clear evidence that neither photoreceptor cells nor monopolar cells contain AChE activity. Kral and Schneider ('81) suggested ACh as transmitter of bee photoreceptors, because they found AChE activity in the extracellular vicinity and also within photoreceptor axons. We used the more direct method of Karnovsky and Roots ('64), which visualizes the esterase directly at the site of enzymatic activity. Because Kral and Schneider ('81) show electron microscope (EM) micrographs obtained after intensification of the staining, it is likely that their method is more prone to artefacts, e.g., the nonenzymatic accretion of crystals. Neither in Drosophila nor in the locust are there any observations on ChAT-LI or AChE activity in photoreceptors (Buchner et al., '86; Lutz and Tyrer, '87). Hardie ('87) proposed histamine as a transmitter of insect photoreceptors on physiological grounds, and we could not demonstrate AChE activity in the photoreceptor. Therefore, it is unlikely that the bee's photoreceptors are cholinergic. The absence of AChE activity in the monopolar cells and their immunoreactivity to an antiserum against glutamate (Bicker et al., '88) suggest that these cells are not cholinergic, but may be glutamatergic. We were able to demonstrate $\alpha$-BTX binding, AChR-LI, and weak but reliable AChE activity in the C-layer of the proximal lamina. The monopolar cells show AChR-LI in their somata and axons, and thus their arborisations may also contribute to the immunoreactivity in the C-layer. These interneurons clearly illustrate that the cellular distribution of AChR-LI is not exactly mirrored by $\alpha$-BTX binding sites. We interpret the fine granular immunoreactivity within the somata of monopolar cells (Fig. 3c) as being an indication of AChR biosynthesis. Neither the cytoplasm nor the membrane of the somata contain $\alpha$-BTX binding sites. Expression of the receptor at the surface of the monopolar cells may, however, cause the simultaneous appearance of AChR-LI and $\alpha$-BTX binding sites (Figs. 3c,d) in the neuropilar arborisations of monopolar cells.

Candidates for providing cholinergic input to monopolar cells are AChE-stained fibers approaching the lamina through the outer chiasma (Fig. 3a). As already pointed out, the chemical neuroanatomy of the lamina and ocellar system closely resemble each other. All sensory cells in the visual and ocellar system display taurine immunoreactivity (Schäfer et al., '88). Their first-order interneurons, the monopolar cells and L-neurons, are glutamate immunoreactive (Bicker et al., '88), and their synaptic contacts are approached by presumably efferent GABA-immunoreactive interneurons (Schäfer and Bicker, '86). This study lists other parallels between the two systems, including AChE activity in amacrine fibers of the lamina and presumably small ocellar interneurons invading the ocellar plexus and AChR-LI concentrated in the C-layer and ocellar plexus. Like the monopolar cells, the large ocellar interneurons do not show AChE activity. Similar to the photoreceptor cells of the compound eye, Kral ('80) described AChE activity in the photoreceptor axons of the ocelli, which directly contradicts our results.

Several of the tracts connecting the optic lobes with the midbrain, and also the commissures interconnecting the two brain hemispheres, contain single fibers with AChE activity (Fig. 4). Besides one of the lobula exits (Fig. 3e), which shows complete staining, most of the optic tracts contain a heterogeneous fiber population with respect to AChE staining.

\section{Antennal and dorsal lobe}

Immunocytochemical studies have suggested various transmitter candidates for interneurons of the antennal and dorsal lobes. GABA immunoreactivity has been demonstrated mainly in local interneurons (Schäfer and Bicker, '86), glutamate immunoreactivity found in projection fibers (Bicker et al., '88), serotonin immunoreactivity in a single deutocerebral giant neuron (Rehder et al., '87), and dopamine immunoreactivity in several identified interneurons (Schäfer and Rehder, '89). The connections between antennal lobe and the mushroom bodies, the median, lateral, and mediolateral antennoglomerular tracts, have especially received the attention of chemoneuroanatomical studies (Bicker et al., '87). The lateral antennoglomerular tract was found to be taurine immunoreactive (Schäfer et al., '88), and the mediolateral antennoglomerular tract contained GABAimmunoreactive fibers (Schäfer and Bicker, '86).

This study describes AChE activity in the magt, which contains interneurons projecting from single glomeruli of the antennal lobe up to the calyces of the mushroom bodies and lateral deutocerebral area. Neither the lagt nor the mlagt showed staining. Labelling of the glomeruli can be explained by AChE activity of magt fibers, although a slight contribution from the antennal receptor terminal cannot be excluded. Degeneration of the receptor fibers, by removal of the antenna, may dissect the contribution of interneurons and sensory afferents to the glomerular AChE staining. Some fibers in the magt showed strong labelling, others intermediate labelling, and a final group remained unlabelled. Mobbs ('85) mentioned that although the antennoglomerular tracts provide a link between antennal lobe and mushroom bodies, they also carry fibers originating in other parts of the brain, for example, the dorsal lobe, tritocerebrum, and sog. The multiple origin of its fibers could therefore lead to a heterogeneous AChE staining of magt fibers.

\section{Mushroom bodies}

Many researchers have stained the projections of the antennoglomerular tracts into the calycal lip area of the mushroom bodies, both by cobalt mass fills from the antennal lobe (Mobbs, '85; Bicker et al., '87) or by intracellular lucifer yellow fills (Homberg, '84). The calyces of the mush-. room bodies contain AChE activity and AChR-LI (Figs. 2, $4 a, 5 a, 6 a, b, d)$, but both properties are pronounced in the lip area (Figs. 2, 6a,b). Therefore, we propose that fibers of the magt send cholinergic projections into the lip, and that the AChR-LI in the lip area represents postsynaptic AChR, which are expressed on the dendrites of the Kenyon cells. The second proposition derives from the observation that somata and fibers of Kenyon cells show AChR-LI. Because the two known projections into the lip neuropile-the antennoglomerular tracts and the protocerebro calycal tract-do not show AChR-LI in their fiber bundles before entering the mushroom bodies, it is rather likely that the AChR-LI in the lip indeed is confined to the Kenyon cells. Because the Kenyon cell somata and their fibers in the lobes and pedunculus are lacking AChE activity, labelling in the calycal neuropile is exclusively of extrinsic origin. We conclude, therefore, that there is no evidence for a cholinergic nature of Kenyon cells, but rather for cholinergic inputs into the various calycal compartments. Some stained fibers running in the anterior superior optic tract and the anterior optic commissure may be responsible for the AChE activity 
in the collar, whereas the source of activity in the basal ring cannot be identified as yet.

\section{Central complex}

Immunocytochemical staining for various transmitter candidates often resulted in a complementary staining pattern of the upper and lower division of the central body. The lower division was, for example, more intensely stained by GABA-immunoreactive fibers than was the upper division (Schäfer and Bicker, '86). Both AChE activity and AChR-LI labelled the lower division stronger than the upper division (Fig. 2), whereas noduli and pons showed a rather homogenous labelling pattern. Thus, the pons is a candidate tract for consisting entirely of cholinergic fiber projections between both hemispheres of the protocerebrum.

\section{Suboesophageal ganglion}

Like the brain, the sog contains AChE and AChR-LI, most of which was difficult to resolve. However, at least some parts of the staining could be attributed to identified anatomical structures. The brain communicates with the thoracic motor centers via descending interneurons, some of which pass through the sog in the MDT. No AChE activity was found in the large diameter fibers of the MDT, which have been reported to be glutamate immunoreactive (Bicker et al., '88). Sensory fibers entering into the sog through the three pairs of mouth nerves showed $\mathrm{AChE}$ staining, whereas the glutamate immunoreactive motoneurons (Bicker et al., '89) were not labelled. Several sensory fibers join the MVT, which also carries the fibers of the tegumentary nerve. The MVT forms a longitudinal sensory tract, which descends through the three neuromeres of the sog into the ventral nerve chord (Rehder, '88). As already discussed, sensory afferents projecting into the antennal lobe, as well as those sensory fibers within the MVT, exhibit strong AChE-LI and therefore may express autoreceptors, too.

In summary, AChE activity was found to be distributed in many tracts and neuropiles of the bee's brain and in a complementary fashion compared with the distribution of GABA, glutamate, and taurine immunoreactivity. This observation lends further support to the assumption that $\mathrm{AChE}$ activity reflects the distribution of cholinergic pathways. Our histochemical investigations provided evidence that the percentage of AChE-stained interneurons is not substantially higher than that of GABA- or glutamateimmunoreactive interneurons (Schäfer and Bicker, '86; Bicker et al., '88). Although ACh is assumed to be the major transmitter in the CNS of insects, most of the cholinergic pathways in the insect brain are probably sensory pathways. This conclusion was also drawn from studies of ChAT immunoreactivity in the locust nervous system (Lutz and Tyrer, '87).

\section{ACKNOWLEDGMENTS}

The authors thank $\mathrm{H}$. Breer for the generous gift of the antiserum against the $\mathrm{ACh}$ receptor, U. Maronde and $\mathrm{R}$. Menzel for helpful discussions, and $\mathrm{M}$. Whitfield for help with the manuscript. This work was supported by a grant of the Deutsche Forschungsgemeinschaft (Bi 262/3-1).

\section{LITERATURE CITED}

Arnold, G., C. Masson, and S. Budharugsa (1985) Comparative study of the antennal lobes and their afferent pathway in the worker bee and the drone (Apis mellifera). Cell Tiss. Res. 242:593-605.

Bicker, G., S. Schäfer, and T. Kingan (1985) Mushroom body feedback interneurones in the honeybee show GABA-like immunoreactivity. Brain Res. 360:394-397.

Bicker, G., S. Schäfer, and V. Rehder (1987) Chemical neuroanatomy of the honeybee brain. In R. Menzel and A. Mercer (eds.): Neurobiology and Behavior of Honeybees. Berlin: Springer-Verlag, pp. 202-224.

Bicker, G., S. Schäfer, O.P. Ottersen, and J. Storm-Mathisen (1988) Glutamate-like immunoreactivity in identified neuronal populations of insect nervous systems. J. Neurosci. 8:2108-2122.

Blagburn, J.M., and D.B. Sattelle (1987) Nicotinic acetylcholine receptors on a cholinergic nerve terminal in the cockroach, Periplaneta americana. J. Comp. Physiol. 161:215-225.

Boeckh, J., K.D. Ernst, H. Sass, and U. Waldow (1984) Anatomical and physiological characteristics of individual neurones in the central antennal pathway of insects. J. Insect Physiol. 30:15-26.

Booth, G.M., and R.L. Metcalf (1970) Phenylthioacetate: A useful substrate for the histochemical and colorimetric detection of cholinesterase. Science 170:455-457.

Booth, G.M., and R.L. Metcalf (1972) The histochemical fate of paraoxon in the cockroach (Periplaneta americana) and honeybee (Apis mellifera) brain. Isr. J. Entomol. 7:143-156.

Breer, H. (1981) Comparative studies on cholinergic activities in the central nervous system of Locusta migratoria. J. Comp. Physiol. 141:271-175.

Breer, H., R. Kleene, and G. Hinz (1985) Molecular forms and subunit structure of the acetylcholine receptor in the central nervous system of insects. J. Neurosci. 5:3386-3392.

Breer, H., and D.B. Sattelle (1987) Molecular properties and functions of insect acetylcholine receptors. J. Insect Physiol. 33:771--790.

Buchner, E., S. Buchner, G. Crawford, W.T. Mason, P.M. Salvaterra, and D.B. Sattelle (1986) Choline acetyltransferase-like immunoreactivity in the brain of Drosophila melanogaster. Cell Tissue Res. 246:57-62.

Buchner, E., and V. Rodrigues (1983) Autoradiographic localization of ${ }^{3} \mathrm{H}$ choline uptake in the brain of Drosophila melanogaster. Neurosci. Lett. 42:25-31.

De Voe, R.D., W. Kaiser, J. Ohm, and L.S. Stone (1982) Horizontal movement detectors of honeybees: Directionally selective visual neurons in the lobula and brain. J. Comp. Physiol. 147:155-170.

Dudai Y. (1980) Cholinergic receptors of Drosophila. In D.B. Sattelle, L.M Hall, and J.G. Hildebrand (eds): Receptors for Neurotransmitters, Hormones and Pheromones in Insects. Amsterdam: Elsevier/North Holland Biomedical Press, pp. 93-110.

Geffard M., A. McRae-Deguerce, and M.L. Souan (1985) Immuncytochemical detection of acetylcholine in the rat central nervous system. Science 229:77-79.

Geffard M., J. Villemaringe, A.-M. Heinrich-Rock, and P. Duris (1985) Antiacetylcholine antibodies and first immunocytochemical application in insect brain. Neurosci. Lett. 57:1-6.

Goodman, L.J. (1981) Organization and Physiology of the insect dorsal ocellar system. In H. Autrum (ed): Handbook of Sensory Physiology. Vol VII/ $6 \mathrm{C}$ Berlin, Heidelberg: Springer, pp. 201-286.

Gorczyca, M.G., and J.C. Hall (1987) Immunohistochemical localization of choline acetyltransferase during development and in Chats mutants of Drosophila melanogaster. J. Neurosci. 7:1361-1369.

Hall, J.C., and D.R. Kankel (1976) Genetics of acetylcholinesterase in Drosophila melanogaster. Genetics 83:517-535.

Hardie, R. (1987) Is histamine a neurotransmitter in insect photoreceptors? J. Comp. Physiol. [A] 161:201-213.

Hertel, H., and U. Maronde (1987) Processing of visual information in the honeybee brain. In R. Menzel and A. Mercer (eds): Neurobiology and Behavior of Honeybees. Berlin: Springer-Verlag, pp. 202-224.

Hertel, H., S. Schäfer, and U. Maronde (1987) The physiology and morphology of visual commissures in the honeybee brain. J. Exp. Biol. 133:283300 .

Hildebrand, J.G., L.M. Hall, and B.C. Osmond (1979) Distribution of binding sites for ${ }^{125}$ I-labelled $\alpha$-bungarotoxin in normal and de-afferented antennal lobes of Manduca sexta. Proc. Natl. Acad. Sci. USA 76:499-503.

Homberg, U. (1984) Processing of antennal information in extrinsic mushroom body neurons in the bee brain. J. Comp. Physiol. [A]154:825-836.

Homberg, U. (1985) Interneurons of the central complex in the beebrain (Apis mellifera L.). J. Insect Physiol. 31:251-264.

Karnovsky, M., and J.L. Roots (1964) A direct coloring method for cholinesterases. J. Histochem. Cytochem. 12:219-222.

Koelle, G.B., and J.S. Friedenwald (1949) A histochemical method for localiz- 
ing cholinesterase activity. Proc. Soc. Exp. Biol. Med. 70:617-622.

Kral, K. (1980) Acetylcholinesterase in the ocellus of Apis mellifica. J. Insect Physiol. 26:807-809.

Kral, K., and L. Schneider (1981) Fine structural localization of acetylcholinesterase activity in the compound eye of the honeybee (Apis mellifica L.). Cell Tiss. Res. 221:351-359.

Levey, A.I., B.H. Wainer, E.J. Mufson, and M.-M. Mesulam (1983) Co-localization of acetylcholinesterase and choline acetyltransferase in the rat cerebrum. Neuroscience 9:9-22.

Lutz, E.M., and N.M. Tyrer (1987) Immunohistochemical localization of choline acetyltransferase in the central nervous system of the locust. Brain Res. 407:173-179.

Matsumoto, S.G., and J.G. Hildebrand (1981) Olfactory mechanisms in the moth Manduca sexta: Response characteristics and morphology of central neurons in the antenrial lobes. Proc. R. Soc. Lond. (Biol.) 213:249277.

Mobbs, P.G. (1985) Brain Structure. In G. Kerkut and L.I. Gilbert (eds): Comprehensive Insect Physiology Pharmacology and Biochemistry, Vol. 5 Nervous Systems: Structure and Motor Function. Oxford: Pergamon Press, pp. 299-370.

Neese, V. (1966) Zur Funktion der Augenborsten bei der Honigbiene. Z. Vergl. Physiol. 49:543-585.

Pareto, A. (1972) Die zentrale Verteilung der Fühlerafferenzen bei Arbeiterinnen der Honigbiene, Apis mellifera L.Z. Zellforsch. 131:109-140.

Pitman, R.M. (1985) Nervous system. In G. Kerkut and L.I. Gilbert (eds): Comprehensive Insect Physiology Pharmacology and Biochemistry, Vol. 11, Pharmacology. Oxford: Pergamon Press, pp. 5-54.

Rehder, V. (1988) A neuroanatomical map of the suboesophageal and prothoracic ganglia of the honey bee (Apis mellifera). Proc. R. Soc. Lond. [Biol] 235:179-202.

Rehder, V., G. Bicker, and M. Hammer (1987) Serotonin-immunoreactive neurons in the antennal lobes and suboesophageal ganglion of the honeybee. Cell Tissue Res. 247:59-66.

Ribi, W.A. (1975) The neurons of the first optic ganglion of the bee (Apis mel- lifera). Adv. Anat. Embryol. Cell Biol. 50:6-45.

Ribi, W.A., and M. Scheel (1981) The second and third optic ganglia in the worker bee. Golgi studies of neuronal elements in the medulla and lobula. Cell Tissue Res. 221:17-43.

Richards, A., and L. Cutkomp (1945) The cholinesterase of insect nerves. J. Cell. Comp. Physiol. 26:57-61.

Rudloff, E. (1978) Acetylcholine receptors in the central nervous system of Drosophila melanogaster. Exp. Cell. Res. 111:185-190.

Salvaterra, P.M., G.D. Crawford, G.D. Klotz, and K. Ikeda (1985) Production and use of monoclonal antibodies to biochemically defined insect neuronal antigens. In H. Beer and T.A. Miller (ed): Neurochemical Techniques in Insect Research. New York: Springer Verlag, pp. 223-242.

Sanes, J.R., and J.G. Hildebrand (1976) Acetylcholine and its metabolic enzymes in developing antennae of the moth, Manduca sexta. Dev. Biol. 52:105-120.

Schäfer S., and G. Bicker (1986) Distribution of GABA- like immunoreactivity in the brain of the honeybee. J. Comp. Neurol. 246:287-300.

Schäfer S., G. Bicker, O.P. Ottersen, and J. Storm-Mathisen (1988) Taurinelike immunoreactivity in the brain of the honeybee. J. Comp. Neurol. 268:60-70.

Schäfer S., and V. Rehder (1989) Dopamine-like immunoreactivity in the brain and suboesophageal ganglion of the honeybee. J. Comp. Neurol. $280: 43-58$.

Schmidt-Nielson, B.K., J.I. Gepner, N.N.H. Teng, and L.M. Hall (1977) Characterization of an alpha-bungarotoxin binding component from Drosophila melanogaster. J. Neurochem. 29:1013-1031.

Sternberger, L.A. (1979) Immunocytochemistry. New York: Wiley.

Suzuki, H. (1975) Antennal movements induced by odor and central projections of the antennal neurons in the honey bee. J. Insect Physiol. 6:168179.

Wang-Bennett, L.T., M.-L. Sovan, and R.M. Glantz (1988) Immuncytochem ical studies of the distribution of acetylcholine in the crayfish brain. J. Comp. Neurol. 273:330-343. 